\title{
PRODUÇÃO E DISTRIBUIÇÃO DE BIOMASSA DE ESPÉCIES ARBÓREAS NO SEMI-ÁRIDO BRASILEIRO ${ }^{1}$
}

\author{
Marcos Antônio Drumond ${ }^{2}$, Ismael Eleotério Pires ${ }^{3}$, Visêldo Ribeiro de Oliveira², Anderson Ramos de \\ Oliveira ${ }^{2}$ Ivan André Alvarez ${ }^{2}$
}

\begin{abstract}
RESUMO - Com o objetivo de avaliar a produção e distribuição de biomassa de algumas espécies arbóreas de múltiplo uso em condições de sequeiro do submédio do São Francisco, instalou-se um experimento no Campo Experimental da Caatinga da Embrapa Semi-Árido, Município de Petrolina, PE. Foram realizadas medições de altura e diâmetro à altura do peito (DAP) de 16 árvores centrais, em três parcelas de cada espécie: Leucaena diversifolia, Caesalpinia velutina, Caesalpinia coriaria, Mimosa tenuiflora e Ateleia herbert-smithii. A biomassa foi estimada com base na árvore de altura média de cada parcela, avaliando-se, separadamente, cada componente (folhas, galhos, cascas e lenho). A biomassa nos diferentes componentes arbóreos das espécies foi distribuída na seguinte ordem: folha<casca<galho<raiz<lenho, excetuando-se A. herbert-smithii, que produziu mais folha do que casca. A produção de biomassa total foi superior em C. velutina $\left(51,6 \mathrm{~kg} \mathrm{ha}^{-1}\right)$, seguida por L. diversifolia (36,6 kg ha-1), A. herbert-smithii (26,4 $\left.\mathrm{kg} \mathrm{ha}^{-1}\right)$, Caesalpinia coriaria $\left(23,0 \mathrm{~kg} \mathrm{ha}^{-1}\right)$ e Mimosa tenuiflora $(21,6$ $\mathrm{kg}$ ha-1). Entretanto, a matéria seca foliar, que é o principal componente forrageiro das espécies, foi maior em C. velutina $\left(3,7 \mathrm{~kg} \mathrm{ha}^{-1}\right)$, vindo em seguida A. herbert-smithii $\left(2,8 \mathrm{~kg} \mathrm{ha}^{-1}\right)$ e $C$. coriaria $\left(2,2 \mathrm{~kg} \mathrm{ha}^{-1}\right), \mathrm{L}$. diversifolia $\left(2,0 \mathrm{~kg} \mathrm{ha}^{-1}\right)$ e $M$. tenuiflora $\left(1,3 \mathrm{~kg} \mathrm{ha}^{-1}\right)$, representando, respectivamente, 7,2; 10,7; 9,5; 5,3; e 6,3\% da biomassa total. C. velutina destacou-se por ser a mais produtiva, tanto como madeireira (lenho=30,8 $\mathrm{t} \mathrm{ha}^{-1}$ ) quanto forrageira (folhas $=3,7 \mathrm{t} \mathrm{ha}^{-1}$ ), enquanto a $M$. tenuiflora foi a de menor potencial forrageiro.
\end{abstract}

Palavras-chave: Forrageiras arbóreas, árvores de uso múltiplo e biomassa.

\section{PRODUCTION AND DISTRIBUTION OF BIOMASS OF TREE SPECIES IN THE BRAZILIAN SEMI-ARID AREA}

\begin{abstract}
The objective of the study was to quantify the production and biomass distribution of some potential arboreal species of multiple uses, under dry land conditions, in the Brazilian Semi-arid. The study was developed in the Experimental Station of Caatinga, of Embrapa Semi-Árido, Petrolina-PE, Brazil. Plant height and diameter at breast height $(\mathrm{DBH})$ of 16 central trees of each plot, from three replicates, were measured. The following species were studied: Leucaena diversifolia, Caesalpinia velutina, Caesalpinia coriaria, Mimosa tenuiflora and Ateleia herbert-smithii. Biomass was estimated based on the medium height tree in each plot, evaluating each component separately: leaf, bark, branch, root and log, except for A. herbert-smithii that produced more leaf than bark. Total biomass production was superior for C. velutina $\left(51.6 \mathrm{~kg} \mathrm{ha}^{-1}\right)$, followed by L. diversifolia $\left(36.6 \mathrm{~kg} \mathrm{ha}^{-1}\right)$, A. herbert-smithii (26.4 $\left.\mathrm{kg} \mathrm{ha}^{-1}\right)$, Caesalpinia coriaria $\left(23.0 \mathrm{~kg} \mathrm{ha}^{-1}\right)$ and Mimosa tenuiflora $\left(21.6 \mathrm{~kg} \mathrm{ha}^{-1}\right.$ However, leaf dry matter, the main potential component of forage species, was higher for C. velutina y A. herbert-smithii

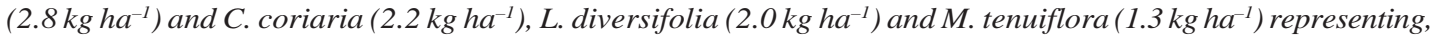
respectively, 7.2, 10.7, 9.5, 5.3 and 6.3\% of total biomass. C. velutina stands out as the most productive, as well as wood $\left(\log =30.8 \mathrm{tha}^{-1}\right)$ as forage (leaves $\left.=3.7 \mathrm{tha}^{-1}\right)$, while M. tenuiflora showed the lowest forage potential.
\end{abstract}

Keywords: Forage trees, multiple use trees and biomass production.

\footnotetext{
${ }^{1}$ Recebido em 26.06.2007 e aceito para publicação em 19.05.2008.

${ }^{2}$ Empresa Brasileira de Pesquisa Agropecuária (EMBRAPA) -Embrapa Semi-Árido, Petrolina-PE. E-mail:<drumond@ cpatsa.embrapa.br>.

${ }^{3}$ Departamento de Engenharia Florestal da Universidade Federal de Viçosa (UFV), Viçosa-MG. E-mail: <iepires@ufv.br>.
} 


\section{INTRODUÇÃO}

O semi-árido brasileiro é considerado a região árida mais habitada do mundo. A formação vegetal presente nessa região é a Caatinga, bioma exclusivamente brasileiro. Contudo, a pressão antrópica sobre esse bioma vem-se intensificando ao longo dos anos, principalmente, a partir do corte indiscriminado de espécies arbóreas nativas.

A instalação de plantios comerciais supre, em parte, a demanda por produtos florestais, reduzindo o corte de espécies nativas. Assim, a introdução de espécies de alta produção de biomassa, adaptadas às condições edafoclimáticas do semi-árido, representa alternativa de renda para os agricultores.

A dependência da população e demais setores da economia em relação ao produto florestal como fonte de energia é muito grande, representando entre $30 \%$ e $50 \%$ da energia primária do Nordeste (CAMPELLO et al., 1999). De acordo com Couto et al. (2000), a biomassa florestal possui características tais que permitem a sua utilização como fonte alternativa de energia, seja na forma de lenha ou de carvão, seja no aproveitamento de resíduos de sua exploração. Esse aspecto importante do manejo de florestas plantadas atende à demanda crescente por matérias-primas para a produção de biomassa e energia para ser utilizada em substituição ao petróleo e seus derivados, buscando-se fontes menos onerosas e renováveis (MALIK et al., 2001; CHHABRA et al., 2002).

Existem espécies nativas da Caatinga que apresentam bom potencial para a produção de carvão vegetal e podem ser consideradas aptas à produção sustentável de biomassa para geração de energia, como é o caso da espécie Mimosa tenuiflora (OLIVEIRA et al., 2006). No entanto, sabe-se que algumas espécies exóticas possuem alto potencial de biomassa, mas não se têm dados concretos sobre a sua produção nas condições do semi-árido brasileiro. Sabe-se que espécies do gênero Leucaena suportam condições de estresse hídrico (LIMA FILHO et al., 1992; PEREZ e FANTI, 1999) como as que ocorrem no bioma Caatinga, além de se apresentarem como forrageiras (MEDINA et al., 2006).

De acordo com Golley et al. (1978), a estimativa da biomassa é um instrumento útil na avaliação de ecossistemas, quanto à sua conversão de energia e ciclagem de nutrientes, absorção e armazenagem de energia solar, possibilitando conclusões para a sua exploração racional. É importante avaliar a produção e distribuição de biomassa das espécies arbóreas de uso múltiplo, visando aumentar a disponibilidade de madeira e forragem na região, e que, indiretamente, contribuam com a preservação das espécies nativas.

Este trabalho teve por objetivo avaliar a produção e distribuição de biomassa de algumas espécies arbóreas introduzidas, de uso múltiplo potenciais para a região semi-árida, em solos de baixa fertilidade, comparado a uma espécie nativa de alto potencial de produção de biomassa.

\section{MATERIAL E MÉTODOS}

O trabalho foi desenvolvido no campo experimental da Embrapa Semi-Árido, Município de Petrolina, PE, em área situada entre as coordenadas geográficas de $09^{\circ} 09^{\prime} \mathrm{S}$ e $40^{\circ} 22^{\prime} \mathrm{W}$. A precipitação média anual varia em torno de $500 \mathrm{~mm}$, concentradas nos meses de fevereiro, março e abril. A temperatura média mensal é de $26^{\circ} \mathrm{C}$, umidade relativa entre 50 e $70 \%$, insolação média de $2.800 \mathrm{~h} /$ ano e evaporação em torno de $2.000 \mathrm{~mm} / \mathrm{ano}$.

As espécies introduzidas foram Leucaena diversifolia (Schld1.) Benth, Caesalpinia velutina (Britton \& Rose) Standley, Caesalpinia coriaria (Jacq.) Willd e Ateleia herbert-smithii Pittier e a espécie nativa Mimosa tenuiflora (Willd.) Poir.

As mudas foram produzidas no viveiro florestal da Embrapa Semi-Árido em sacos de polietileno preto com dimensões de $8 \mathrm{~cm}$ x $12 \mathrm{~cm}$ e transferidas para o campo aos 60 dias após o semeio com altura média de $25 \mathrm{~cm}$.

O delineamento estatístico utilizado foi em blocos ao acaso com cinco tratamentos e três repetições, com espaçamento de $3 \times 2 \mathrm{~m}$. Cada espécie foi considerada um tratamento, sendo a espécie nativa referenciada como testemunha. Foram utilizadas parcelas quadradas constituídas de 36 plantas e avaliadas as 16 centrais quanto a altura, diâmetro à altura do peito (DAP) e sobrevivência (\%). Para a estimativa da produção de biomassa foi selecionado um indivíduo de porte médio de cada parcela, conforme Drumond et al. (1997).

Em cada parcela foram coletadas amostras simples do solo nas profundidades de 0 a 20, 20 a 40 e 40 a 
$60 \mathrm{~cm}$, posteriormente submetidas às análises dos atributos físicos e químicos de rotina.

As avaliações foram realizadas quando as espécies estavam com a idade de 8 anos. Os parâmetros avaliados foram: biomassa das raízes, profundidade da raiz principal, número de raízes secundárias e raio de ocupação do sistema radicular. A obtenção dos parâmetros foi feita por meio da retirada do sistema radicular de cada indivíduo de porte médio, por espécie, em cada parcela.

A avaliação da parte aérea foi feita separadamente por componente: folhas, galhos, cascas e lenho.

\section{RESULTADOS E DISCUSSÃO}

O índice de sobrevivência das mudas de todas as espécies foi bom (maior que 75\%), considerando e que durante a condução do experimento não se utilizou irrigação. A avaliação da sobrevivência não demonstrou diferenças que fossem significativas entre as espécies. Observou-se que as espécies C. velutina e L. diversifolia se destacaram das demais quanto à altura (Quadro 1).

Quadro 1 - Comportamento silvicultural de algumas espécies arbóreas de uso múltiplo na região semi-árida do Nordeste brasileiro, aos 8 anos de idade

Table 1 - Silvicultural behavior of some arboreal species of multiple uses in the semi-arid region of the Brazilian Northeast, after eight years planting date

\begin{tabular}{lccc}
\hline \multicolumn{1}{c}{ Espécie } & $\begin{array}{c}\text { Sobrevivência } \\
(\%)\end{array}$ & $\begin{array}{c}\text { Altura } \\
(\mathrm{m})\end{array}$ & $\begin{array}{c}\text { Diâmetro } \\
(\mathrm{cm})\end{array}$ \\
\hline Ateleia herbert-smithii & $77 \mathrm{a}$ & $4,4 \mathrm{~b}$ & $4,5 \mathrm{~b}$ \\
Caesalpinia coriaria & $100 \mathrm{a}$ & $3,0 \mathrm{c}$ & $3,3 \mathrm{c}$ \\
Caesalpinia vel utina & $100 \mathrm{a}$ & $5,8 \mathrm{a}$ & $5,6 \mathrm{a}$ \\
Leucaena diversifolia & $78 \mathrm{a}$ & $5,9 \mathrm{a}$ & $4,5 \mathrm{~b}$ \\
Mimosa tenuiflora & $81 \mathrm{a}$ & $3,7 \mathrm{bc}$ & $3,4 \mathrm{c}$ \\
\hline
\end{tabular}

Médias seguidas da mesma letra, na coluna, não diferem entre si, pelo teste de Tukey a $5 \%$ de probabilidade.

C. velutina apresentou melhor comportamento, em relação à altura, que as outras espécies, com índice de sobrevivência de $100 \%$ e DAP de $5,6 \mathrm{~cm}$, sendo este último, estatisticamente, superior aos demais. Esses resultados, de maneira geral, são superiores quando comparados com os apresentados por Drumond et al. (1999), para as espécies nativas de sucesso na região: Anadenanthera macrocarpa, Schinopis brasiliensis e Mimosa caesalpiniifolia, que, com a mesma idade, apresentaram alturas de 4,0; 5,0; e 3,0 e DAP de 4,0; 5,0; e 4,0 cm, respectivamente, e nas outras espécies exóticas, Leucaena leucocephala e Prosopis juliflora, com alturas de 5,0 e 5,0 m e DAP de 5,0 e 6,0 cm, respectivamente. Observase, ainda, que a espécie nativa $M$. tenuiflora apresentou diâmetro e altura inferiores à $C$. velutina $\mathrm{e} L$. diversifolia .

A produção de biomassa total para C. velutina $\left(51,64 \mathrm{t} \mathrm{ha}^{-1}\right)$ foi superior à das demais espécies (Quadro 2), embora não diferenciando estatisticamente de $L$. diversifolia $\left(36,59 \mathrm{t} \mathrm{ha}^{-1}\right)$ e A. herbert-smithii $(26,41$ $\left.\mathrm{t} \mathrm{ha}^{-1}\right)$. Entretanto, porcentualmente a biomassa foliar foi maior em A. herbert-smithii (10,7\%), seguida da C. coriaria $(9,5 \%)$, o que indica serem espécies com características de grande potencial forrageiro.

Considerando que espécies exóticas, de maneira geral, apresentam menor número de inimigos naturais selecionados, elas podem apresentar vantagem competitiva quanto às características silviculturais. Dessa forma, plantios dirigidos à exploração de sua biomassa são considerados uma excelente opção para que a Caatinga seja preservada. Entretanto, sabe-se que a introdução de novas espécies em um ecossistema pode vir a causar problemas. Há sempre o risco de ela se tornar espécie invasora.

A distribuição da biomassa nos diferentes componentes arbóreos das espécies seguiu, assim, esta ordem: folha<casca<galho<raiz<lenho, exceto em $M$. tenuiflora e C. coriaria que produziram mais galho do que raiz; e A. herbert-smithii, que produziu mais folha do que casca (Quadros 2 e 3). Resultados semelhantes foram encontrados por Moura et al. (2006), que observaram que a biomassa seca dos diferentes componentes arbóreos em um povoamento de Mimosa caesalpiniifolia foi distribuída na seguinte ordem: lenho > galhos > casca $>$ folhas, corroborando resultados dos trabalhos de Drumond et al. (1996), nas espécies Newtonia contorta (DC.) Burkart e Endlicheria paniculata (Spreng.) Macbr.

Notou-se que a espécie nativa $M$. tenuiflora diferencia-se das demais por alocar para a copa $42,8 \%$ da biomassa aérea total, sendo $36,5 \%$ desta relativa aos galhos, justificando o elevado número de bifurcações e galhos, característica específica de espécies nativa da Caatinga. Esses resultados concordam com aqueles obtidos por Drumond e Oliveira (1998) em Mimosa tenuiflora (Willd.). Portanto, a espécie nativa tem maior capacidade de adaptação, comparando-se com as espécies introduzidas de sobrevivência nas condições inóspitas do semi-árido.

R. Árvore, Viçosa-MG, v.32, n.4, p.665-669, 2008 
Quadro 2 - Biomassa seca e sua distribuição porcentual (entre parênteses) nos componentes da parte aérea de algumas espécies arbóreas de uso múltiplo na região semi-árida do Nordeste brasileiro, aos 8 anos de idade

Table 2 - Dry biomass and its percentual distribution (in parentheses) in the upper part of some arboreal species for multiple uses in the semi-arid region of Northeast Brazil, eight years after planting

\begin{tabular}{lccccccc}
\hline \multirow{2}{*}{ Espécie } & $\begin{array}{c}\text { Folha } \\
(\mathrm{F})\end{array}$ & $\begin{array}{c}\text { Galho } \\
(\mathrm{G})\end{array}$ & $\begin{array}{c}\text { Copa } \\
(\mathrm{F}+\mathrm{G})\end{array}$ & $\begin{array}{c}\text { Casca } \\
(\mathrm{C})\end{array}$ & $\begin{array}{c}\text { Lenho } \\
(\mathrm{L})\end{array}$ & $\begin{array}{c}\text { Tronco } \\
(\mathrm{C}+\mathrm{L})\end{array}$ & $\begin{array}{c}\text { Total } \\
\text { Ateleia herbert-smithii }\end{array}$ \\
\cline { 2 - 8 } & $2,8 \mathrm{~A}$ & $5,5 \mathrm{~A}$ & $8,3 \mathrm{~A}$ & $2,2 \mathrm{~B}$ & $15,9 \mathrm{AB}$ & $18,1 \mathrm{AB}$ & $26,4 \mathrm{AB}$ \\
& $(10,7)$ & $(20,9)$ & $(31,6)$ & $(8,1)$ & $(60,3)$ & $(68,4)$ & $(100)$ \\
Caesal pinia coriaria & $2,2 \mathrm{~A}$ & $7,9 \mathrm{~A}$ & $10,1 \mathrm{~A}$ & $2,3 \mathrm{~B}$ & $10,6 \mathrm{~B}$ & $12,9 \mathrm{~B}$ & $23,0 \mathrm{~B}$ \\
& $(9,5)$ & $(34,6)$ & $(44,1)$ & $(9,9)$ & $(46,0)$ & $(55,9)$ & $(100)$ \\
Caesal pinia vel utina & $3,7 \mathrm{~A}$ & $11,6 \mathrm{~A}$ & $15,3 \mathrm{~A}$ & $5,5 \mathrm{~A}$ & $30,8 \mathrm{~A}$ & $36,3 \mathrm{~A}$ & $51,6 \mathrm{~A}$ \\
& $(7,2)$ & $(22,5)$ & $(29,7)$ & $(10,6)$ & $(59,7)$ & $(70,3)$ & $(100)$ \\
Leucaena diversifolia & $2,0 \mathrm{~A}$ & $8,5 \mathrm{~A}$ & $10,5 \mathrm{~A}$ & $3,8 \mathrm{AB}$ & $22,3 \mathrm{AB}$ & $26,1 \mathrm{AB}$ & $36,6 \mathrm{AB}$ \\
& $(5,3)$ & $(23,3)$ & $(28,6)$ & $(10,4)$ & $(61,0)$ & $(71,4)$ & $(100)$ \\
Mimosa tenuiflora & $1,3 \mathrm{~A}$ & $7,9 \mathrm{~A}$ & $9,2 \mathrm{~A}$ & $3,8 \mathrm{AB}$ & $8,6 \mathrm{~B}$ & $12,4 \mathrm{~B}$ & $21,6 \mathrm{~B}$ \\
& $(6,3)$ & $(36,5)$ & $(42,8)$ & $(17,5)$ & $(39,7)$ & $(57,2)$ & $(100)$ \\
\hline
\end{tabular}

Médias seguidas da mesma letra, na coluna, não diferem entre si, pelo teste de Tukey a $5 \%$ de probabilidade.

Quadro 3 - Dados de crescimento do sistema radicular de algumas espécies arbóreas de uso múltiplo na região semi-árida do Nordeste brasileiro, aos 8 anos de idade

Table 3 - Data on growth of root system of some arboreal species of multiple uses in the semi-arid region of the Brazilian Northeast, after eight years planting date

\begin{tabular}{lcccccc}
\hline \multirow{2}{*}{ Espécie } & $\begin{array}{c}\text { Biomas sa parte } \\
\text { aérea }\end{array}$ & Biomassa raiz & $\begin{array}{c}\text { Relação } \\
\text { R/PA }\end{array}$ & Comprimento raiz & $\begin{array}{c}\mathrm{N}^{2} \text { de } \\
\text { raízes }\end{array}$ & $\begin{array}{c}\text { Raio de projeção } \\
\text { hori zontal da raiz }\end{array}$ \\
\cline { 2 - 6 } & t/ha & t/ha & - & $\mathrm{m}$ & $\mathrm{un}$ \\
\hline A. herbert-smithii & 26,41 & 11,52 & 0,43 & 0,90 & 17 & 1,2 \\
C. coriaria & 22,99 & 7,60 & 0,33 & 1,20 & 1,5 \\
C. velutina & 51,64 & 12,04 & 0,23 & 0,95 & 1,1 \\
L. diversifolia & 36,59 & 9,18 & 0,25 & 0,70 & 1,1 \\
M. tenuiflora & 21,62 & 6,60 & 0,30 & 1,00 & 9 & 1,0 \\
\hline
\end{tabular}

PA - Parte aérea.

A espécie $C$. velutina apresentou grande capacidade de formação de parte aérea $\left(51,6 \mathrm{tha}^{-1}\right)$ com reduzida biomassa de raiz (12 $\left.\mathrm{t} \mathrm{ha}^{-1}\right)$, enquanto na espécie $A$. herbert-smithii a relação biomassa da parte aérea e biomassa da raiz foi menor. Isso indica que a biomassa da raiz e a biomassa da parte aérea desenvolvem-se em proporções variadas entre cada espécie (Figura 1).

\section{CONCLUSÕES}

A Caesalpinia velutina foi a espécie que mostrou melhor crescimento, considerando-se as variáveis: altura, diâmetro, sobrevivência e biomassa da parte aérea.

R. Árvore, Viçosa-MG, v.32, n.4, p.665-669, 2008

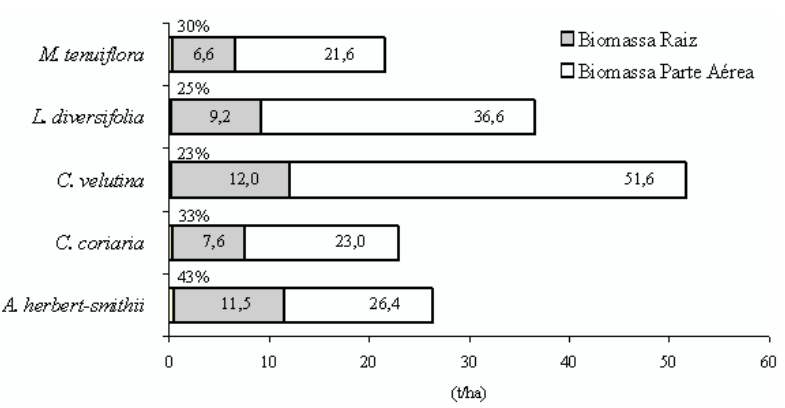

Figura 1 - Biomassa produzida e relação entre a raiz e a parte aérea de algumas espécies arbóreas introduzidas na região semi-árida do Nordeste do Brasil, aos 8 anos de idade.

Figure 1 - Produced biomass and relation between the root system and the upper part of some arboreal species introduced in the semi-arid region of the Brazilian Northeast, after eight years planting date. 
A distribuição da biomassa nos diferentes componentes da planta das espécies estudadas obedeceu à seguinte ordem: folha<casca < galho<raiz<lenho, exceto em $M$. tenuiflora e $C$. coriaria, que produziram mais galho do que raiz; e $A$. herbert-smithii, que geraram mais folha do que casca.

\section{REFERÊNCIAS}

CAMPELLO, F.B.; GARIGLIO, M.A.; SILVA, J.A.; LEAL, A.M.A. Diagnóstico florestal da região Nordeste. Brasília: IBAMA/PNUD, 1999.

CHHABRA, A.; PALRIA, S; DADHWAL, V.K. Growing stock-based forest biomass estimate for India. Biomass and Bioenergy, v. 22, n.3, p.187-194, 2002.

COUTO, L.; FONSECA, E.M.B.; MÜLLER, M.D. O estado da arte das plantações de florestas de rápido crescimento para produção de biomassa para energia em Minas Gerais: aspectos técnicos, econômicos sociais e ambientais. Belo Horizonte: CEMIG, 2000.

DRUMOND, M.A.; BARROS, N.F.; SILVA, A.F. Composição florística e fitossociológica de uma mata secundária de um trecho da Mata Atlântica na região do Médio Rio Doce - MG. In: CONGRESSO NACIONAL DE BOTÂNICA, 48., 1997, Crato. Resumos... Crato: Sociedade Brasileira de Botânica - SBB, 1997.

DRUMOND, M.A.; OLIVEIRA, V.R.; CARVALHO FILHO, O.M. Introdução e seleção de espécies forrageiras exóticas na região semi-árida do estado de Sergipe. Acta Botanica

Brasilica, v.13, n.3, p.251-256, 1999.

DRUMOND, M.A.; OLIVEIRA, V.R. Introducción y selección de especies arbóreas forrajeras en el area Semiárida del Estado de Pernambuco, Brasil. In: CONGRESO LATINOAMERICANO IUFRO. EL MANEJO SUSTENTABLE DE LOS RECURSOS FLORESTALES, DESAFIO DEL SIGLO TEMA4, 1., 1998, Valdivia. Anais... Valvidia: IUFRO/ CONAF, 1998. 1 CD-ROM.
DRUMOND, M.A.; BARROS, N.F.; SOUZA, A.L.; SILVA, A.L.; MEIRA NETO, J.A.A. Alterações fitossociológicas e edáficas na Mata Atlântica em função das modificações da cobertura vegetal. Rev. Árvore, v.20, p.451-466, 1996.

GOLLEY, F.B., McGINNIS, J.T.; CLEMENTS, R.G.; CHILD, G.I.; DUEVER, M.J. Ciclagem de minerais em um ecossistema de floresta tropical úmida. São Paulo: EDUSP, 1978.

LIMA FILHO, J.M.P.; DRUMOND, M.A.; MACENO, D.S. Comportamento fisiológico de Leucena e Albizia sob condições semi-áridas. Pesq. agropec. bras., Brasília, v.27, n.4, p.537- 542, 1992.

MALIK, R.K.; GREEN, T.H.; BROWN, G.F.; BEYL, C.A.; SISTANI, K.R.; MAYS, D.A. Biomass production of short-rotation bioenergy hardwood plantations affected by cover crops. Biomass and Bioenergy, v.21, n.1, p. 21-33, 2001.

MEDINA, J.F.J.; PINTO, R.R.; HERNÁNDEZ, L.A.; ALVARADO, L.; GÓMEZ, C.H.; MARTÍNEZ, C.B.; NAHED, T.J.; CARMONA, J. Produccion de fitomasa de especies arboreas $y$ arbustivas de uso forrajero en el valle central de Chiapas, Mexico. México, 2004. Disponível em: <http://www.lead.virtualcentre.org/ es/enl/keynote8.htm>. Acesso em: 14 abr. 2006.

MOURA, O.N.; PASSOS, M.A.A.; FERREIRA, R.L.C.; MOLICA, S.G.; LIRA JUNIOR, M.A.; LIRA, M.A.; SANTOS, M.V.F. Distribuição de biomassa e nutrientes na área de Mimosa caesalpiniaefolia Benth. Rev. Árvore, v.30, n.6, p.877-884, 2006.

OLIVEIRA, E.; VITAL, B.R.; PIMENTA, A.S.; LUCIA, R.M.D.; LADEIRA, A.M.; CARNEIRO, A.C.O. Estrutura anatômica da madeira e qualidade do carvão de Mimosa tenuiflora (Willd.) Poir. Rev. Árvore, v.30, n.2, p.311-318, 2006.

PEREZ, S.C.J.G.A.; FANTI, S.C. Crescimento e resistência à seca de leucena em solo de cerrado. Pesquisa Agropecuária Brasileira, Brasília, v. 34, n. 6, p.933-944, 1999

R. Árvore, Viçosa-MG, v.32, n.4, p.665-669, 2008 